Ann. Biol. anim. Bioch. Biophys., 1979, 19 (2 B), 543-549.

\title{
C. Influence de l'huile de colza très paurre en acide érucique sur la reproduction de la poule
}

\author{
par B. LECLERCQ, M.-R. SALICHON
}

Station de Recherches Avicoles, I.N.R.A.,

Nouzilly, 37380 Monnaie.

Summary. Effect of low-erucic acid rapeseed oil on the reproduction of hens.

During 4 trials, high-erucic acid and low-erucic acid (Primor oil) rapeseed oils were compared to peanut oil after 8 p. 100 incorporation into the diet of laying hens. Contrary to the high-erucic acid rapeseed oil, the low-erucic acid oil did not change egg or yolk weight or the frequency of ovipositions (laying rate). However, in 3 trials, embryonic mortality increased significantly when viability was good in the control lot.

La poule pondeuse est un animal particulièrement sensible à la nature des hulies incorporées à son aliment. Ce phénomène a été très étudié à propos de l'huile de maïs et des autres sources d'acide linoléique (Calvert, 1965). Mais on sait aussi que d'autres graisses peuvent exercer des effets qui sont, eux, défavorables. II en est ainsi des huiles contenant des acides gras de forme trans (Leclercq, Blum et Jacquot, 1965 ; Leclercq et Blum, 1967). Dès 1968, plusieurs équipes de recherches ont montré que c'était également le cas des huiles de colza (Kondra, Choo et Sell, 1968 ; Leclercq, 1968 ; Chudy ef al., 1969) qui, à des doses faibles, réduisent significativement le poids de l'œuf, et à des doses plus importantes (plus de 10 p. 100) entraînent une baisse significative du rythme d'ovipositions (taux de ponte). Ces effets de l'huile de colza semblent dus essentiellement à la présence d'acide érucique dans l'huile (Leclercq, 1970 ; Leclercq, 1972). Il est donc apparu intéressant d'utiliser cet animal particulièrement sensible que constitue la poule en ponte, pour comparer l'huile de Primor à d'autres huiles alimentaires y compris l'huile de colza riche en acide érucique.

\section{Matériel et méthodes.}

L'ensemble de l'étude comporte 4 essais. Les animaux utilisés sont des Warrens qui servent à plusieurs essais successifs après une phase de réhabilitation au cours de laquelle tous les animaux reçoivent un même aliment. Ainsi, le premier essai est réalisé avec un premier lot de poules et les 3 suivants avec un second lot. L'âge et le nombre des animaux seront précisés à l'occasion de chaque essai. Les poules sont 
élevées en batteries de cages individuelles dans des chambres thermostatées à $20^{\circ} \mathrm{C}$; elles sont réparties en un certain nombre de répétitions constituant des blocs au sein desquels figure un représentant de chaque lot expérimental. Les aliments sont fabriqués à partir d'un mélange complémentaire identique pour tous, auquel sont adjoints 8 p. 100 de l'huile à tester. Un premier mélange vitaminique a été utilisé au cours du premier essai. Il était calculé de façon à ne couvrir que les besoins de ponte, aucun test de fertilité et d'éclosivité n'ayant été prévu lors de la conception de cet essai : on n'a pas pris en considération les besoins vitaminiques correspondants. II n'en a pas été de même dans les 3 derniers essais, où un second mélange vitaminique, mieux adapté aux besoins des animaux pour l'ensemble des tests étudiés, a été substitué au premier.

TABLEAU 1

Composition du mélange de base

\begin{tabular}{|c|}
\hline 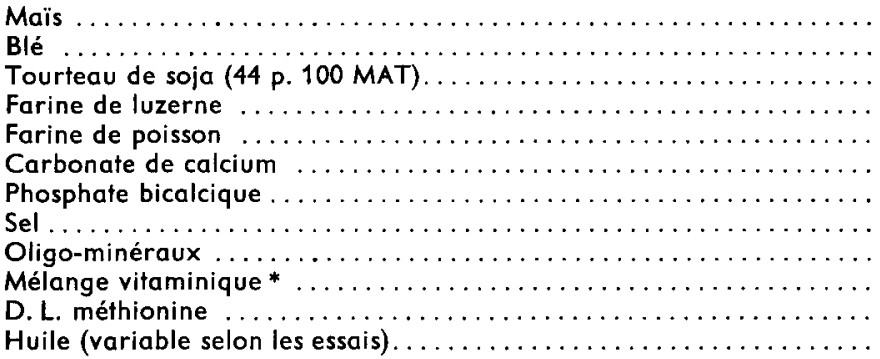 \\
\hline
\end{tabular}

* Composition du mélange vitaminique (pour $100 \mathrm{~kg}$ ) :

Mélange 1 : Vitamine A : $800000 \mathrm{UI}$; Vitamine $D_{3}: 100000 \mathrm{UI}$; Vitamine $\mathrm{E}: 4 \mathrm{~g}$; BHT : $10 \mathrm{~g}$; Pantothénate de calcium : $0,5 \mathrm{~g}$; Niacine : $1 \mathrm{~g}$; Pyridoxine : $0,1 \mathrm{~g}$.

Mélange 2 : Vitamine A : $1000000 \mathrm{UI}$; Vitamine $D_{3}: 150000 \mathrm{UI}$; Vitamine $\mathrm{E}: 1,5 \mathrm{~g}$; BHT : $12,5 \mathrm{~g}$; Ménadione : $0,5 \mathrm{~g}$; Riboflavine : $0,4 \mathrm{~g}$; Pantothénate de calcium : $0,8 \mathrm{~g}$; Niacine $: 2,5 \mathrm{~g}$; Pyridoxine : $0,1 \mathrm{~g}$; Cyanocobalamine : $0,8 \mathrm{mg}$; Acide folique : $20 \mathrm{mg}$; Biotine : $20 \mathrm{mg}$; Choline : $50 \mathrm{~g}$.

Le mélange 1 a été utilisé lors du 1 er essai, le mélange 2 lors des trois autres.

Les huiles comparées lors du premier essai ont été fournies par l'ITERG en 1975 et sont communes aux essais entrepris à cette époque sur d'autres espèces. Les huiles des trois derniers essais provenaient des Etablissements Robbe (Compiègne) pour l'huile de lin et l'huile de soja brutes, des huileries Noel (Pont-Saint-Esprit) pour l'huile de colza Primar. Les tests d'incubation destinés à mesurer d'éventuels effets sur le développement embryonnaire ont été réalisés après insémination des poules avec $100 \mu \mathrm{l}$ d'un mélange de spermes non dilués provenant d'une dizaine de coqs.

Essai $n^{0} 1$. - Trois lots de 20 poules âgées de 34 semaines reçoivent un même aliment du commerce au cours de la période préexpérimentale qui dure 4 semaines. Les régimes expérimentaux sont distribués au cours de l'essai proprement dit qui s'étend sur 6 semaines. Les comparaisons portent sur les variations observées entre 
période préexpérimentale et période expérimentale. Trois huiles sont comparées : I'huile d'arachide (lot témoin), l'huile de colza riche en acide érucique et l'huile de colza Primor. En fin d'essai, un test de fertilité et d'éclosivité est réalisé sur 150 œufs de chaque lot.

Essai $n^{0} 2$. - Cet essai est entrepris afin de comparer les effets de l'huile de colza Primor et de l'huile d'arachide à ceux d'un mélange d'huile de lin (1,2 p. 100) ef d'huile d'arachide (6,8 p. 100) apportant la même quantité d'acide linolénique. Un quatrième lot reçoit de l'huile de soja brute. Les taux d'incorporation de l'huile sont de 8 p. 100. Chaque lot comporte 36 poules. L'essai dure 56 jours. Les animaux ont 24 semaines au début de l'essai et serviront aux essais 3 et 4 . Une incubation d'œufs est réalisée avec $\mathbf{2 8 0}$ œufs de chaque lot.

Essai no 3. - Les résultats d'incubation des essais 1 ef 2 étant en contradiction, on entreprend l'essai 3 qui permet de comparer l'huile d'arachide et l'huile de colza Primor avec l'un ou l'autre des mélanges vitaminiques utilisés dans l'essai 1 ou l'essai 2 ; soif un plan factoriel $2 \times 2$ combinant le facteur " huile » et le facteur " mélange vitaminique ». Chacun des 4 lots renferme 18 poules. L'essai dure 28 jours après une phase d'adaptation aux régimes de 14 jours. On fait incuber 150 œufs de chacun des lots.

Essai no 4. - L'objectif de ce dernier essai est de comparer l'huile de Primor de l'essai no 1 à l'huile de Primor des essais suivants. L'huile d'arachide représente le témoin. A cette occasion, l'huile de lin brute est également comparée aux huiles. Chacune est distribuée à 18 poules. L'essai dure 46 jours (15 jours d'adaptation ef 31 jours d'expérience proprement dite).

TABLEAU 2

Performances des poules pondeuses selon la nafure de l'huile ingérée. (Essai no 1)

\begin{tabular}{|c|c|c|c|}
\hline & Préexpérience & Expérience & Différence \\
\hline $\begin{array}{l}\text { Taux de ponte }(p .100) \ldots \ldots \ldots \ldots \ldots \ldots \ldots \\
\quad \begin{array}{c}\text { Huile d'arachide } \ldots \ldots \ldots \ldots \ldots \ldots \ldots \\
\text { Huile de colza } \ldots \ldots \ldots \ldots \ldots \ldots\end{array} \\
\text { Huile de Colza Primor } \ldots \ldots \ldots \ldots \ldots\end{array}$ & $\begin{array}{l}81,9 \\
87,3 \\
83,2\end{array}$ & $\begin{array}{l}82,8 \\
78,1 \\
80,3\end{array}$ & $\begin{array}{r}+0,9 a \\
-9,2 b \\
-2,9 a\end{array}$ \\
\hline $\begin{array}{l}\text { Poids moyen de l'œuf }(g) \ldots \ldots \ldots \ldots \ldots \ldots \\
\quad \begin{array}{l}\text { Huile d'arachide } \ldots \ldots \ldots \ldots \ldots \ldots \ldots \\
\text { Huile de colza } \ldots \ldots \ldots \ldots \ldots \ldots\end{array} \\
\text { Huile de colza Primor } \ldots \ldots \ldots \ldots \ldots\end{array}$ & $\begin{array}{l}61,8 \\
61,1 \\
61,9\end{array}$ & $\begin{array}{l}63,8 \\
57,8 \\
63,5\end{array}$ & $\begin{array}{r}+2,1 \mathrm{a} \\
-3,3 \mathrm{~b} \\
+1,6 \mathrm{a}\end{array}$ \\
\hline 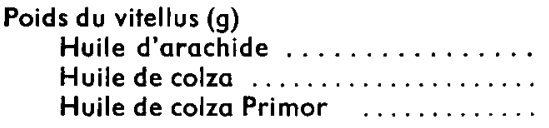 & $\begin{array}{l}17,7 \\
17,8 \\
17,8\end{array}$ & $\begin{array}{l}18,4 \\
14,9 \\
17,7\end{array}$ & $\begin{array}{r}+0,7 a \\
-2,9 b \\
-0,1 a\end{array}$ \\
\hline $\begin{array}{l}\text { Consommation d'aliment }(\mathrm{g} / \mathrm{j}) \\
\text { Huile d'arachide } \ldots \ldots \ldots \ldots \ldots \ldots \ldots \ldots \\
\text { Huile de colza } \ldots \ldots \ldots \ldots \ldots \ldots \ldots \\
\text { Huile de colza Primor } \ldots \ldots \ldots \ldots \ldots\end{array}$ & & $\begin{array}{l}124,6 \\
117,2 \\
118,9\end{array}$ & \\
\hline
\end{tabular}

Les différences accompagnées d'une même lettre ne sont pas significativement différentes au seuil de 5 p. 100. Les moyennes ont été comparées selon le test de Newman et Keuls. 


\section{Résultats.}

Les résultats du premier essai font l'objet du tableau 2. L'huile de colza riche en acide érucique réduit le taux de ponte, le poids moyen de l'œuf et celui du vitellus, alors que les huiles de colza Primor ef d'arachide permettent des performances identiques. Les résultats du test d'éclosion sont rapportés dans le tableau 3. L'huile de colza riche en acide érucique augmente significativement la morfalité embryonnaire (†est $\chi^{2}$ à 5 p. 100) par rapport à l'huile d'arachide. Il en est de même de l'huile de colza Primor, même par rapport aux deux autres huiles (test $\chi^{2}$ à 1 p. 100). La mortalité survenant entre 18 jours et l'éclosion est significativement différente au seuil de 1 p. 100 entre chacun des lots expérimentaux.

TABLEAU 3

Fertilité et éclosivité des œufs issus de poule ayant ingéré différentes huiles. (Essai $n^{0} 1$ )

\begin{tabular}{|c|c|c|c|c|c|}
\hline & $\begin{array}{l}\text { Nombre } \\
\text { d'œufs } \\
\text { incubés }\end{array}$ & $\begin{array}{l}\text { Nombre } \\
\text { d'œufs } \\
\text { infertiles }\end{array}$ & $\begin{array}{l}\text { Embryons } \\
\text { morts de } \\
4 \text { à } 18 j\end{array}$ & $\begin{array}{l}\text { Embryons } \\
\text { morts à } \\
\text { l'éclosion }\end{array}$ & $\begin{array}{c}\text { Poussins } \\
\text { éclos }\end{array}$ \\
\hline $\begin{array}{l}\text { Huile d'arachide } \ldots \ldots \ldots \ldots \ldots \\
\text { Huile de colza............... } \\
\text { Huile de colza Primor } \ldots \ldots \ldots\end{array}$ & $\begin{array}{l}152 \\
147 \\
143\end{array}$ & $\begin{array}{r}10 \\
3 \\
18\end{array}$ & $\begin{array}{r}9 \\
21 \\
65\end{array}$ & $\begin{array}{r}5 \\
29 \\
48\end{array}$ & $\begin{array}{r}128 \\
94 \\
12\end{array}$ \\
\hline
\end{tabular}

Les essais suivants ont été entrepris pour vérifier et tenter d'expliquer les effets inattendus et surprenants de l'huile de colza Primor. L'essai no 2 a pour but de savoir si l'acide linolénique de l'huile de colza Primor est responsable de la mortalité embryonnaire. Une nouvelle huile de colza Primor est donc comparée à l'huile d'arachide (lot témoin), à un mélange d'huile d'arachide el d'huile de lin apportant la même quantité d'acide linolénique que l'huile de colza Primor et à de l'huile de soja contenant, elle aussi, des quantités appréciables du triène. Les résultats de cet essai font l'objet du tableau 4. Le taux de ponte et le poids moyen de l'œuf ne sont pas modifiés par la

TABLEAU 4

Performances de ponte des poules en fonction de la nature de l'huile ingérée et résultats d'incubation des œufs. (Essai no 2)

\begin{tabular}{|c|c|c|c|c|}
\hline & $\begin{array}{c}\text { Huile } \\
\text { d'arachide }\end{array}$ & $\begin{array}{c}\text { Huile de } \\
\text { colza Primor }\end{array}$ & $\begin{array}{c}\text { Huile de lin } \\
+ \\
\text { Huile d'arachide }\end{array}$ & $\begin{array}{l}\text { Huile } \\
\text { de soja }\end{array}$ \\
\hline $\begin{array}{l}\text { Taux de ponte }(\mathrm{p} .100) \ldots \ldots \ldots \ldots \\
\text { Poids moyen de l'œuf }(\mathrm{g}) \ldots \ldots \ldots \ldots \\
\text { Incubation }\end{array}$ & $\begin{array}{l}91,4 \\
55,0\end{array}$ & $\begin{array}{l}90,2 \\
53,7\end{array}$ & $\begin{array}{l}94,3 \\
54,0\end{array}$ & $\begin{array}{l}91,7 \\
55,3\end{array}$ \\
\hline $\begin{array}{l}\text { Infertilité (p. 100) .............. } \\
\text { Mortalité embryonnaire (p. 100) } \\
\text { Eclosivité (p. 100) ........... }\end{array}$ & $\begin{array}{l}6,0 \\
6,8 a \\
87,2 a\end{array}$ & $\begin{array}{c}7,4 \\
8,9 b \\
83,7 b\end{array}$ & $\begin{array}{c}9,9 \\
10,6 b \\
79,5 \mathrm{~b}\end{array}$ & $\begin{array}{c}8,1 \\
9,2 \mathrm{~b} \\
82,7 \mathrm{~b}\end{array}$ \\
\hline
\end{tabular}


nature de l'huile ingérée. Les seules différences significatives concernent l'éclosivité qui est plus faible pour les œufs provenant de poules ayant ingéré soit l'huile de colza Primor, soit le mélange d'huile de lin et d'huile d'arachide, soit l'huile de soja. Cette réduction est toutefois beaucoup plus faible que celle qui a été constatée dans l'essai précédent.

L'essai no 3 est entrepris pour étudier l'influence de l'huile de colza Primor en fonction du mélange vitaminique, ce dernier ayant été largement complété afin de tenir compte des besoins particuliers liés au développement embryonnaire. On pouvait envisager en effet une interaction entre huile et vitamines due, par exemple, à la présence d'une substance à activité antivitaminique dans l'huile de colza Primor. Nous présentons les résultats de cet essai dans le tableau 5 . Ni l'huile, ni le mélange

\section{TABLEAU 5}

Influence de la nature de l'huile et de la composition du mélange vitaminique sur les performances de ponte et les résultats d'incubation des œufs. (Essai $n^{0} 3$ ).

\begin{tabular}{|c|c|c|c|c|}
\hline & \multicolumn{2}{|c|}{ Huile d'arachide } & \multicolumn{2}{|c|}{ Huile de colza Primor } \\
\hline & $\begin{array}{l}\text { Mélange } \\
\text { vitaminique } \\
n^{\circ} 2\end{array}$ & $\begin{array}{c}\text { Mélange } \\
\text { vitaminique } \\
n^{\circ} 1\end{array}$ & $\begin{array}{l}\text { Mélange } \\
\text { vitaminique } \\
n^{\circ} 2\end{array}$ & $\begin{array}{l}\text { Mélange } \\
\text { vitaminique } \\
n^{\circ} 1\end{array}$ \\
\hline $\begin{array}{l}\text { Taux de ponte }(p .100) \ldots \ldots \ldots \ldots \\
\text { Poids moyen de l'œuf }(g) \ldots \ldots \ldots \\
\text { Incubation }\end{array}$ & $\begin{array}{l}88,3 \\
58,5\end{array}$ & $\begin{array}{l}86,1 \\
57,7\end{array}$ & $\begin{array}{l}83,5 \\
58,1\end{array}$ & $\begin{array}{l}83,7 \\
59,1\end{array}$ \\
\hline $\begin{array}{l}\text { Infertilité (p. 100) } \ldots \ldots \ldots \ldots \ldots \\
\text { Mortalité embryonnaire }(p .100) \\
\text { Eclosivité }(p .100) \ldots \ldots \ldots \ldots\end{array}$ & $\begin{array}{r}9,9 \\
3,9 a \\
85,5 a\end{array}$ & $\begin{array}{l}11,3 \\
27,6 \mathrm{c} \\
61,1 \mathrm{c}\end{array}$ & $\begin{array}{r}14,9 \\
9,0 \mathrm{~b} \\
76,1 \mathrm{~b}\end{array}$ & $\begin{array}{l}7,9 \\
27,3 \mathrm{c} \\
64,8 \mathrm{c}\end{array}$ \\
\hline
\end{tabular}

vitaminique n'exerce d'effet significatif sur les performances de ponte. II en va tout autrement de la mortalité embryonnaire qui est diminuée de façon spectaculaire selon la supplémentation en vitamines. Quant à l'huile de colza Primor, elle n'augmente cette mortalité par rapporł à l'huile d'arachide que lorsque l'apport vitaminique est élevé. Ces effets se traduisent par des réductions semblables de l'éclosivité.

TABLEAU 6

Influence de la nature de l'huile sur les performances de ponte ef les résulfats d'incubation des æufs. (Essai no 4)

\begin{tabular}{|c|c|c|c|c|}
\hline & $\begin{array}{c}\text { Huile } \\
\text { d'arachide }\end{array}$ & $\begin{array}{l}\text { Huile } \\
\text { de lin }\end{array}$ & $\begin{array}{l}\text { Huile de } \\
\text { colza Primor } \\
\text { de l'essai } 1\end{array}$ & $\begin{array}{c}\text { Huile de } \\
\text { colza Primor } \\
\text { des essais } 2 \text { et } 3\end{array}$ \\
\hline $\begin{array}{l}\text { Taux de ponte }(p .100) \ldots \ldots \ldots \ldots \\
\text { Poids moyen de l'œuf }(g) \ldots \ldots \ldots \ldots \\
\text { Incubation }\end{array}$ & $\begin{array}{l}85,7 \\
60,7\end{array}$ & $\begin{array}{l}85,3 \\
59,9\end{array}$ & $\begin{array}{l}86,2 \\
60,4\end{array}$ & $\begin{array}{l}83,3 \\
59,9\end{array}$ \\
\hline $\begin{array}{l}\text { Infertilité (p. 100) } \ldots \ldots \ldots \\
\text { Mortalité embryonnaire (p. 100) } \\
\text { Eclosivité (p. 100) } \ldots \ldots \ldots \ldots\end{array}$ & $\begin{array}{r}13,6 \\
9,7 \\
76,8\end{array}$ & $\begin{array}{r}11,1 \\
7,0 \\
81,9\end{array}$ & $\begin{array}{r}17,6 \\
5,6 \\
76,8\end{array}$ & $\begin{array}{r}14,3 \\
9,7 \\
760\end{array}$ \\
\hline
\end{tabular}


On réalise l'essai no 4 pour comparer l'huile de colza Primor de l'essai no 1 à celle des essais no 2 et 3 . En effet, au cours de ces deux derniers essais, l'éclosivité des lots recevant l'huile de colza Primor n'est pas aussi réduite que lors de l'essai nº 1 , ce qui laisse supposer que les huiles n'étaient pas semblables. Le tableau 6 contient les résultats de ce dernier essai auquel nous avons adjoint un lot recevant 8 p. 100 d'huile de lin, la bibliographie ne fournissant guère d'information sur l'influence de cefte huile sur la reproduction de la poule pondeuse. Aucune différence significative ne peut être observée entre les lots, même pour ce qui concerne l'éclosivité.

\section{Discussion et conclusion.}

Les résultats des quatre essais présentés ci-dessus confirment un certain nombre d'observations antérieures. Il est clairement montré tout d'abord que la présence d'acide érucique dans l'huile de colza réduit la taille de l'œuf et en particulier du jaune ; l'huile de colza Primor très pauvre en cet acide gras n'a pas cet effet. Nous confirmons donc nos premières observations réalisées sur l'huile de canbra (Leclercq, 1970) et renouvelées ensuite par Lall et Slinger (1973). Des conclusions similaires peuvent être faites à propos du taux de ponte dans l'essai no 1.

Les essais suivants où ne figure plus l'huile de colza riche en acide érucique, mais seulement l'huile de colza Primor, confirment bien l'innocuité de cette dernière.

Si les conclusions relatives au poids moyen de l'œuf et au taux de ponte sont suffisamment nettes et conformes à ce que l'on prévoit, il n'en est pas de même pour les effets sur le développement embryonnaire et l'éclosivité. Nous avons observé en 1971 (Leclercq et Edwards, données non publiées) que l'huile de colza riche en acide érucique réduit l'éclosivité en augmentant la mortalité embryonnaire. Lall et Slinger (1973) confirment bien ce résultat : plus l'huile est riche en acide érucique, ou plus importante est son incorporation à l'aliment, plus le p. cent d'œufs qui éclosent est diminué. Cette observation est renouvelée au cours de l'essai no 1 . Mais ce sont les résultats concernant l'huile de colza Primor qui surprennent au cours de cet essai. Ceux-ci sont très inférieurs à ceux obtenus avec l'huile de colza classique. De plus, Lall et Slinger ne trouvent pas d'effet dépressif à l'huile de colza pauvre en acide érucique $(2,2$ p. 100) sur l'éclosivité. Les essais 2 et 3 confirment bien cet effet, mais de façon très atténuée. L'essai no 2 rendrait d'ailleurs vraisemblable l'hypothèse d'une responsabilité de l'acide linolénique. Quant à l'essai no 3, il montre que l'action dépressive de l'huile de colza Primor n'est perceptible que lorsque le reste du régime, en particulier l'apport de vitamines, est satisfaisant. Le dernier essai vient remettre en question les conclusions précédentes : les huiles de colza Primor ne réduisent alors plus significativement l'éclosivité. L'huile de colza Primor dont l'influence avait été particulièrement prononcée au cours de l'essai no 1 ne diffère plus ni de l'huile de colza Primor des essais suivants, ni de l'huile d'arachide. L'huile de lin, elle-même distribuée à raison de 8 p. 100 du régime, ne manifeste aucun effet défavorable au développement embryonnaire.

L'intervention de l'huile de colza Primor dans le développement embryonnaire du poussin paraît cependant être réelle. Elle a été mise en évidence au cours de trois essais sur quatre. Ceux-ci ont tous été entrepris de façon à s'assurer que les différences observées proviennent uniquement de l'huile : répartition équilibrée des animaux, 
constitution rigoureuse des régimes, insémination des poules au hasard ef non pas par régime. On doit donc suspecter une interaction entre l'huile ef un autre facteur. Celuici peut être un constituant de régime. Il faut noter aussi que l'effet dépressif du colza Primor est surtout évident quand l'éclosivité du lot témoin recevant de l'huile d'arachide est élevée.

Quoi qu'il en soit, comme les autres huiles de colza très pauvres en acide érucique, l'huile de colza Primor n'affecte pas les performances de reproduction de la poule (poids de l'œuf, du vitellus et taux de ponte). Il existerait cependant une substance autre que l'acide érucique et responsable de la mortalité embryonnaire observée assez fréquemment, aussi bien avec l'huile de colza classique qu'avec l'huile de colza Primor. Ce peut très bien être un composé non lipidique de la graisse entraîné lors de l'extraction de l'huile. L'essai no 1 laisserait suspecter une substance à activité antiriboflavine; la riboflavine du régime couvre en effet le besoin de ponte, mais pas celui de reproduction. Cependant, l'essai no 3 ne confirme pas cette hypothèse qui nécessiterait, pour être vérifiée, la mise en œuvre des dosages de cette vitamine dans les matières premières. Ce peut être aussi un herbicide ou un insecticide ou un produit dérivé présent sur la graine ef extrait avec les lipides, un phénomène semblable ayant déjà été observé avec l'huile de soja (Mounts ef al., 1976).

Effets physiologiques comparés des huiles de colza et d'arachide. ATP-INRA, 1974-1976.

\section{Références}

CALVERT C. C., 1965. Lipid metabolism in the hen : essential fatty acids. Proc. Cornell Nutr. Conf. Feed Manuf., 104-112.

CHUDY J., JAWORSKA H., JAWORSKY J., RUTKOWSKIA, 1969. The influence of fat contained in fodder mixtures on fatty acid composition in hens eggs. Rocz. Tech. Chem. Zyw., 16, 61-69.

KONDRA P. A., CHOO S. H., SELL J. L., 1968. Influence of strain of chicken and dietary fat on egg production traits. Poult. Sci., 47, 1290-1296.

LALL S. P., SLINGER S. J., 1973. Nutritional evaluation of rapessed oils and rapeseed soapstocks for laying hens. Poult. Sci., 52, 1729-1740.

LECLERCQ B., 1968. Utilisation de l'huile de colza par la poule pondeuse ; son influence sur la genèse et la composition des lipides de l'œuf. C. R. Acad. Sci., Paris, sér. D, 267, 2235-2237.

LECLERCQ B., 1970. Facteurs nutritionnels modifiant le poids de l'œuf et de constituants. Ann. Biol. anim. Bioch. Biophys., 10, 239-252.

LECLERCQ B., 1972. Influence de l'acide érucique alimentaire chez la poule pondeuse : diminution du poids de l'œuf. Ann. Biol. anim. Bioch. Biophys., 12, 505-508.

LECLERCQ B., BLUM J. C., 1967. Utilisation de l'huile d'arachide isomérisée par la poule pondeuse. Ses effets sur la genèse et la composition de réserves vitellines. Ann. Biol. anim. Bioch. Biophys., 7, 303-315.

LECLERCQ B., BLUM J. C., JACQUOT R., 1965. Ełude de l'utilisation de l'acide élaïdique au cours de l'ovogenèse de Gallus gallus : son incorporation dans les graisses de réserves et dans les lipides de l'œuf pondu. C. R. Acod. Sci., 261, 3197-3200.

MOUNTS T. L., DUTTON H. J., EVANS C. D., COWAN J. C., 1976. Chick edema factor : removal from soybean oil. J. amer. Oil Chem. Soc., 53, 105-107. 